\title{
A meta-analysis of the medium- and long- term effects of laparoscopic sleeve gastrectomy and laparoscopic Roux-en-Y gastric bypass
}

$\mathrm{Lihu} \mathrm{Gu}^{1+}$, Xiaojing Huang ${ }^{2+}$, Shengnan $\mathrm{Li}^{2}$, Danyi Mao ${ }^{3}$, Zefeng Shen ${ }^{4}$, Parikshit Asutosh Khadaroo ${ }^{5}$, Derry Minyao $\mathrm{Ng}^{6}$ and Ping Chen ${ }^{1 *}$

\begin{abstract}
Background: Laparoscopic Roux-en-Y gastric bypass (LRYGB) and laparoscopic sleeve gastrectomy (LSG) are two representative bariatric surgeries. This study aimed to compare the effects of the LSG and LRYGB based on highquality analysis and massive amount of data.

Methods: For this study databases of PubMed, Web of Science, EBSCO, Medline, and Cochrane Library were searched for articles published until January 2019 comparing the outcomes of LSG and LRYGB.

Results: This study included 28 articles. Overall, 9038 patients (4597, LSG group; 4441, LRYGB group) were included. The remission rate of type 2 diabetes mellitus (T2DM) in the LRYGB group was superior to that in the LSG group at the 3-years follow-up. Five-year follow-up results showed that LRYGB had an advantage over LSG for the percentage of excess weight loss and remission of T2DM, hypertension, dyslipidemia, and abnormally low-density lipoprotein.
\end{abstract}

Conclusions: In terms of the long-term effects of bariatric surgery, the effect of LRYGB was better than of LSG.

Keywords: Sleeve gastrectomy, Roux-en-Y gastric bypass, Effects, Meta-analysis

\section{Background}

Worldwide, obesity not only seriously affects the external appearance but also causes various diseases, which threaten people's health. The usual way to lose weight is through dieting or medication use, which reduces weight by 5 to $10 \%$. However, the resulting weight loss is short term, leading to rebound weight gain. Nowadays, bariatric surgery is widely known, as it has long-lasting effectiveness $[1,2]$. Meanwhile, it can also alleviate some complications [3].

Laparoscopic Roux-en-Y gastric bypass (LRYGB) and laparoscopic sleeve gastrectomy (LSG) are two representative bariatric surgeries. The origin of LRYGB can be

\footnotetext{
*Correspondence: chenpinghwamei@163.com

'Lihu Gu and Xiaojing Huang contributed equally to this work.

'Department of General Surgery, HwaMei Hospital, University of Chinese

Academy of Sciences, Northwest Street 41, Haishu District, Ningbo, Zhejiang

315010, China

Full list of author information is available at the end of the article
}

traced back to about 50 years ago. As LRYGB has excellent effectiveness on alleviating obesity complications, including type 2 diabetes (T2DM), it is known as the gold standard surgery for obese patients [4-8]. However, impaired micronutrient absorption is more common after LRYGB [9-11]. Some studies had shown that patients treated with LRYGB were more likely to have vitamin B12 deficiency after surgery than patients treated with LSG, but there was no difference in the absorption of folic acid and the effect on serum iron was controversial [11-14].

Moreover, conclusions of studies that compared LRYGB with LSG in the remission of complications remained controversial. Some studies indicated that LRYGB was superior to LSG $[5,15,16]$ in the remission of T2DM, while other studies suggested that the remission rates were similar in both groups [17]. Previous studies had shown an advantage for LRYGB in the 
remission of hypertension [18-20]. With these differences in results, this study aimed to scientifically compare the advantages of LRYGB and LSG based on highquality analysis and massive amount of data.

\section{Methods}

\section{Literature search}

This meta-analysis was in line with the recommendations of Preferred Reporting Items for Systematic Reviews and Meta-Analyses (PRISMA) statement [21]. Electronic literature search was conducted from inception to January 2019 of various databases including PubMed, EMBASE, Cochrane library, Web of Science, and EBSCO. The following keywords were used: ("laparoscopic Roux-en-Y gastric bypass" OR "gastric bypass" OR "GB" OR "LRYGB") AND ("laparoscopic sleeve gastrectomy" OR "sleeve gastrectomy" OR "SG" OR "LSG"). Two researchers separately performed the literature search and compared their results. Most of the articles were screened manually by scanning titles and abstracts. Then, through a further check, all initially included studies were downloaded finally.

\section{Inclusion and exclusion criteria}

The primary outcomes of the studies included in the analysis were percentage of excess weight loss (\%EWL) and remission rates of T2DM. The secondary outcomes of the studies were remission rates of hypertension and dyslipidemia. Published studies comparing the outcomes of LSG and LRYGB were considered potentially eligible. The follow-up time was at least 3 years. The exclusion criteria were as follows: (1) non-original article; (2) results did not include \%EWL and remission rates of T2DM, hypertension, and dyslipidemia; and (3) no available medium-term (3-year) or long-term (5-year) original data or relevant outcome. Any unclear data were deleted decisively, and we made sure that all data were checked more than twice.

\section{Data extraction and quality assessment}

After multiple inspections, a list of articles that finally met the inclusion criteria was created, and a reviewer extracted the following basic indicators from each article (Table 1): study country and year, sample size, follow-up time, number of patients who completed the final follow-up, and study type. The indicators of \%EWL and remissions of T2DM, hypertension, and dyslipidemia in each article were extracted.

The quality of included articles was evaluated using the Cochrane risk-of-bias tool and the NewcastleOttawa Quality Assessment Scale (NOS) checklist [22, 23]. According to the recommendations of the Cochrane manual, the risk of bias in randomized controlled trial (RCTs) is categorized as low risk, unclear risk, and high risk. Observational studies were assessed by the NOS. Each article was evaluated in three aspects as follows: object selection, inter-group comparability, and outcome measurement. Articles with scores $<6$ were considered low-quality articles. Any contradiction between reviewers was resolved by the consensus of two authors and the third reviewer.

\section{Statistical analysis}

RevMan 5.3 software was used to integrate statistical results. Weighted mean difference (WMD) was used to collect continuous variables, while odds ratios (OR) was used to analyze dichotomous variables. Heterogeneity was checked by Cochrane Collaboration's risk of tool, chi-squared test, and $\mathrm{I}^{2}$ statistics and identified when $p<0.1$ and $\mathrm{I}^{2}>50 \%$. If the results were heterogeneous, a random-effects model was used to calculate the combined effect size; otherwise, a fixed-effects model was used. The Stata 12.0 Software (Stata, College Station) was used to evaluate the sensitivity and publication bias of the studies. Publication bias was evaluated by Begg's and Egger's tests, and $p<0.05$ was considered statistically significant. Begg's and Egger's tests of publication bias were not performed to analyze subgroups with less than 10 articles because of the low sensitivity of qualitative and quantitative tests. All statistical tests were twosided, and $p<0.05$ was considered statistically significant.

\section{Results}

The PRISMA flowchart of literature search is shown in Fig. 1. The initial database search retrieved 9482 articles. No article was found through other sources. After removing 5314 duplicates, 4015 publications for nonsurgical procedures were excluded. Among the 153 publications that met our criteria, 3 were excluded as they did not compare LSG and LRYGB, 22 were non-original articles, and 21 had no available original data. The follow-up of 52 studies was less than 3 years, and 27 articles had no outcomes relevant to our bariatric surgery. Therefore, 28 articles were included in analysis $[1-7,10$, $11,14,15,17-20,24-36]$. After serious consideration, all articles were of great research value and could provide strong evidence for our meta-analysis. Countries included were very representative and vast, including Chile, China, Finland, France, Greece, India, Italy, Lebanon, Netherlands, Poland, Singapore, Spain, Switzerland, USA, and Venezuela.

\section{Study characteristics}

In total, the characteristics of the 28 studies are shown in Table 1.The included studies consist of seven RCTs, six prospective observational studies, and 15 retrospective observational studies. Overall, 9038 patients (4597 in 
Table 1 Characteristics of studies included in the meta-analysis

\begin{tabular}{|c|c|c|c|c|c|c|c|c|}
\hline \multirow[t]{2}{*}{ Author, year } & \multirow[t]{2}{*}{ Country } & \multicolumn{2}{|c|}{$\begin{array}{l}\text { No. of } \\
\text { participants }\end{array}$} & \multirow{2}{*}{$\begin{array}{l}\text { Follow- } \\
\text { up } \\
\text { (year) }\end{array}$} & \multicolumn{2}{|c|}{$\begin{array}{l}\text { No. of } \\
\text { remaining }\end{array}$} & \multirow[t]{2}{*}{ Comorbidities remission (without medication) } & \multirow[t]{2}{*}{ Study type } \\
\hline & & $\overline{\mathrm{LSG}}$ & $\overline{L R Y G B}$ & & $\overline{\mathrm{LSG}}$ & LRYGB & & \\
\hline Abbatini, 2010 & Italy & 20 & 16 & 3 & 20 & 16 & $\mathrm{FPG}<126 \mathrm{mg} / \mathrm{dl}, \mathrm{HbA} 1 \mathrm{c}<6.5 \%$ & Retrospective \\
\hline Ahmed, 2018 & USA & 59 & 57 & 7 & 27 & 26 & NA & Prospective \\
\hline $\begin{array}{l}\text { Alexandrou, } \\
2014\end{array}$ & Greece & 40 & 55 & 4 & 40 & 55 & NA & Prospective \\
\hline $\begin{array}{l}\text { Dakour Aridi, } \\
2018\end{array}$ & Lebanon & 400 & 175 & 5 & 87 & 118 & NA & Retrospective \\
\hline Boza, 2012 & Chile & 811 & 786 & 3 & 811 & 786 & $\mathrm{FPG}<126 \mathrm{mg} / \mathrm{dl}, \mathrm{HbA} 1 \mathrm{c}<6.5 \%$ & Retrospective \\
\hline $\begin{array}{l}\text { Carandina, } \\
2014\end{array}$ & France & 34 & 74 & 4 & 34 & 74 & NA & Retrospective \\
\hline Dogan, 2015 & Netherlands & 255 & 430 & 5 & 245 & 245 & NA & Retrospective \\
\hline Du, 2016 & China & 63 & 63 & 3 & 60 & 59 & $\mathrm{FPG}<5.6 \mathrm{mmol} / \mathrm{l}, \mathrm{HbA} 1 \mathrm{c}<6 \% / \mathrm{BP}<120 / 80 \mathrm{mmHg}$ & Retrospective \\
\hline Climent, 2018 & Spain & 48 & 103 & 5 & 48 & 103 & NA & Retrospective \\
\hline $\begin{array}{l}\text { Gonzalez- } \\
\text { Heredia, } 2016\end{array}$ & USA & 77 & 12 & 3 & 30 & 8 & NA & Retrospective \\
\hline Ignat, 2017 & France & 55 & 45 & 5 & 41 & 32 & NA & $\mathrm{RCT}$ \\
\hline Jammu, 2016 & India & 339 & 295 & 5 & 97 & 143 & NA & Prospective \\
\hline Jimenez, 2012 & Spain & 55 & 98 & 3 & 55 & 98 & $\mathrm{FPG}<126 \mathrm{mg} / \mathrm{dl}, \mathrm{HbA} 1 \mathrm{c}<6.5 \%$ for at least 1 year & Prospective \\
\hline Kim, 2019 & Singapore & 256 & 39 & 3 & 71 & 10 & NA & Retrospective \\
\hline Kaseja, 2014 & Poland & 33 & 41 & 3 & 33 & 41 & NA & Prospective \\
\hline Lager, 2018 & USA & 334 & 380 & 4 & 226 & 272 & $\mathrm{HbA} 1 \mathrm{c}<6.5 \% / \mathrm{BP}<120 / 80 \mathrm{mmHg}$ & Retrospective \\
\hline Lee, 2015 & China & 519 & 519 & 5 & 116 & 218 & NA & Retrospective \\
\hline Leyba, 2014 & Venezuela & 42 & 75 & 5 & 27 & 47 & $\mathrm{HbA} 1 \mathrm{c}<6 \%$ & Prospective \\
\hline Perrone, 2017 & Italy & 162 & 142 & 5 & 162 & 142 & NA & Retrospective \\
\hline Peterli, 2018 & Switzerland & 112 & 113 & 5 & 101 & 104 & $\mathrm{FPG}<100 \mathrm{mg} / \mathrm{dl}, \mathrm{HbA} 1 \mathrm{c}<6.0 \%$ at least 1 year & $\mathrm{RCT}$ \\
\hline Rondelli, 2017 & Italy & 280 & 301 & 3 & 259 & 282 & NA & Retrospective \\
\hline $\begin{array}{l}\text { Ruiz-Tovar, } \\
2019\end{array}$ & Spain & 200 & 200 & 5 & 182 & 184 & $\begin{array}{l}\mathrm{FPG}<110 \mathrm{mg} / \mathrm{dl}, \mathrm{HbA} 1 \mathrm{c}<6.5 \% / \mathrm{BP}<135 / 85 \mathrm{mmHg} / \mathrm{FPT}<200 \mathrm{mg} / \\
\mathrm{dl}, \mathrm{TC}<200 \mathrm{mg} / \mathrm{dl}, \mathrm{HDL}>40 \mathrm{mg} / \mathrm{dl}\end{array}$ & $\mathrm{RCT}$ \\
\hline Salminen, 2018 & Finland & 121 & 119 & 5 & 98 & 95 & $\mathrm{FPG}<100 \mathrm{mg} / \mathrm{dl}, \mathrm{HbA} 1 \mathrm{c}<6.0 \% / \mathrm{LDL}<115.8 \mathrm{mg} / \mathrm{dl}$ & $\mathrm{RCT}$ \\
\hline $\begin{array}{l}\text { Sepulveda, } \\
2018\end{array}$ & Chile & 57 & 55 & 3 & 41 & 35 & $\mathrm{FPG}<100 \mathrm{mg} / \mathrm{dl}, \mathrm{HbA} 1 \mathrm{c}<6.0 \%$ & Retrospective \\
\hline Vidal, 2013 & Spain & 114 & 135 & 4 & 91 & 108 & NA & Retrospective \\
\hline Yang, 2015 & China & 32 & 32 & 3 & 28 & 27 & $\mathrm{HbA} 1 \mathrm{c}<6.0 \%$ & $\mathrm{RCT}$ \\
\hline Zhang, 2014 & China & 32 & 32 & 5 & 26 & 28 & NA & $\mathrm{RCT}$ \\
\hline Schauer, 2017 & USA & 47 & 49 & 5 & 47 & 49 & $\mathrm{HbA} 1 \mathrm{c}<6.5 \%$ & $\mathrm{RCT}$ \\
\hline
\end{tabular}

LSG laparoscopic sleeve gastrectomy; $L R Y G B$ laparoscopic Roux-en-Y gastric bypass; NA no available; FPG fasting plasma glucose; HbA1c glycosylated hemoglobin; $B P$ blood pressure; FPT fasting plasma triglycerides; $T C$ total cholesterol; $L D L$ low-density lipoprotein; $H D L$ high-density lipoprotein; $R C T$ Randomized clinical trial

the LSG group, 4441 in the LRYGB group) were included. All of them were followed for at least 3 years, and 13 studies of them were followed for 5 years or longer. The results of assessment of quality and risk of bias for all included studies were included in Additional file 1: Table S1.

$\%$ EWL

$\% \mathrm{EWL}$ is an essential metric that measures the effect of weight loss after bariatric surgery. A total of 19 articles reported \%EWL after surgery. Among them, 13 articles provided 3-year follow-up data, 9 articles provided 5year follow-up data, and 3 articles provided both 3-year and 5-year data. At 3-year follow-up, \%EWL in the LRYGB group was greater than that in the LSG group $(\mathrm{WMD}=-4.37, \quad 95 \% \mathrm{Cl}=-8.10-(-0.64), \quad p=0.02$, random-effects model). Subgroup analysis performed according to the type of study revealed that the postoperative effect of LRYGB group was better than that of LSG group in RCTs (WMD $=-11.96,95 \% \mathrm{Cl}=-17.62-(-$ 


\section{श्रISMA PRISMA 2009 Flow Diagram}

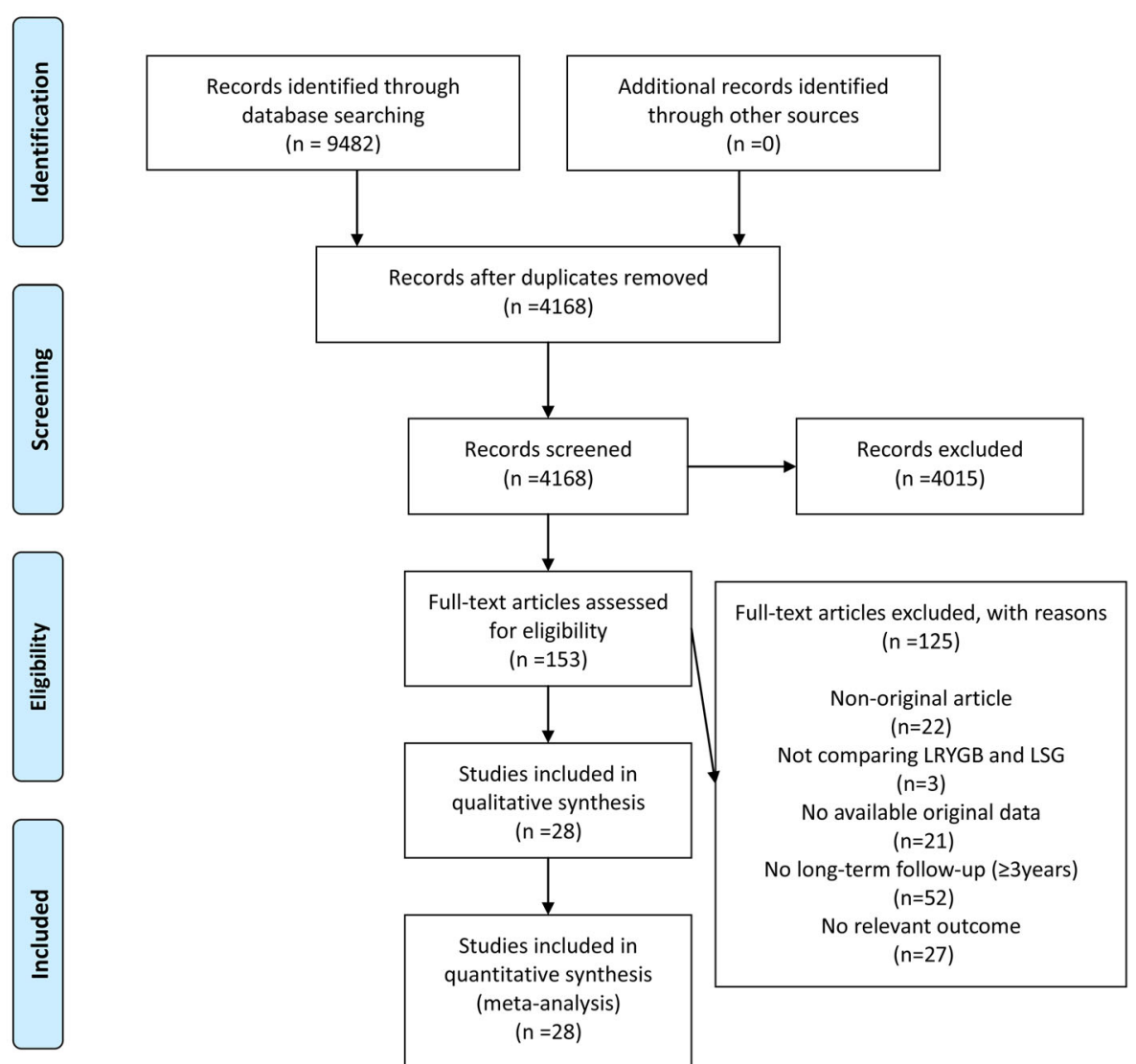

Fig. 1 Flow diagram of study inclusion and exclusion

6.30), $p<0.001)$. Moreover, no significant difference in the treatment effect between the two groups was found, in either prospective or retrospective studies (Fig. 2).

Comparison of the outcome of \%EWL in the fifth postoperative year showed that patients who underwent LRYGB had greater \%EWL than those who underwent LSG (WMD $=-2.20,95 \% \mathrm{Cl}=-3.83-(-0.57), p=0.008$, random-effects model). Subgroup analysis of LSG and LRYGB in 5-year follow-up revealed that the LRYGB group has better outcomes than the LSG group in both retrospective studies (WMD $=-2.05,95 \% \mathrm{Cl}=-2.60-(-$ 1.50), $p<0.001$ ) and RCTs (WMD $=-6.36,95 \% \mathrm{Cl}=-$ 12.51-(-0.20), $p=0.04$ ) (Fig. 2).

\section{Resolution of obesity-related comorbidities}

Many studies investigated the improvement of obesityrelated comorbidities during the postoperative period. Some publications discussed comorbidities such as arthritis, obstructive sleep apnea, hyperuricemia, and depression. This meta-analysis detected the effect of remission of only hypertension, T2DM, and dyslipidemia.

\section{T2DM}

Of the 28 articles, 14 mentioned remission rates of T2DM representing 1018 patients (490 in the LSG group, 528 in the LRYGB group). The remission rate of T2DM in the LRYGB group was higher than that in the LSG in 3-years follow-up $(\mathrm{OR}=0.68,95 \% \mathrm{CI}=$ $0.48-0.95, p=0.02$, fixed-effects model), so was the remission rate in 5-year follow-up $(\mathrm{OR}=0.63,95 \%$ $\mathrm{CI}=0.41-0.96, \quad p=0.03$, fixed-effects model). Subgroup analysis according to the type of study revealed that the remission rate in LRYGB was higher than that in LSG in prospective studies with 3-year followup $(\mathrm{OR}=0.46,95 \% \mathrm{CI}=0.24-0.89, \quad p=0.02)$. In addition, no significant difference was found between 


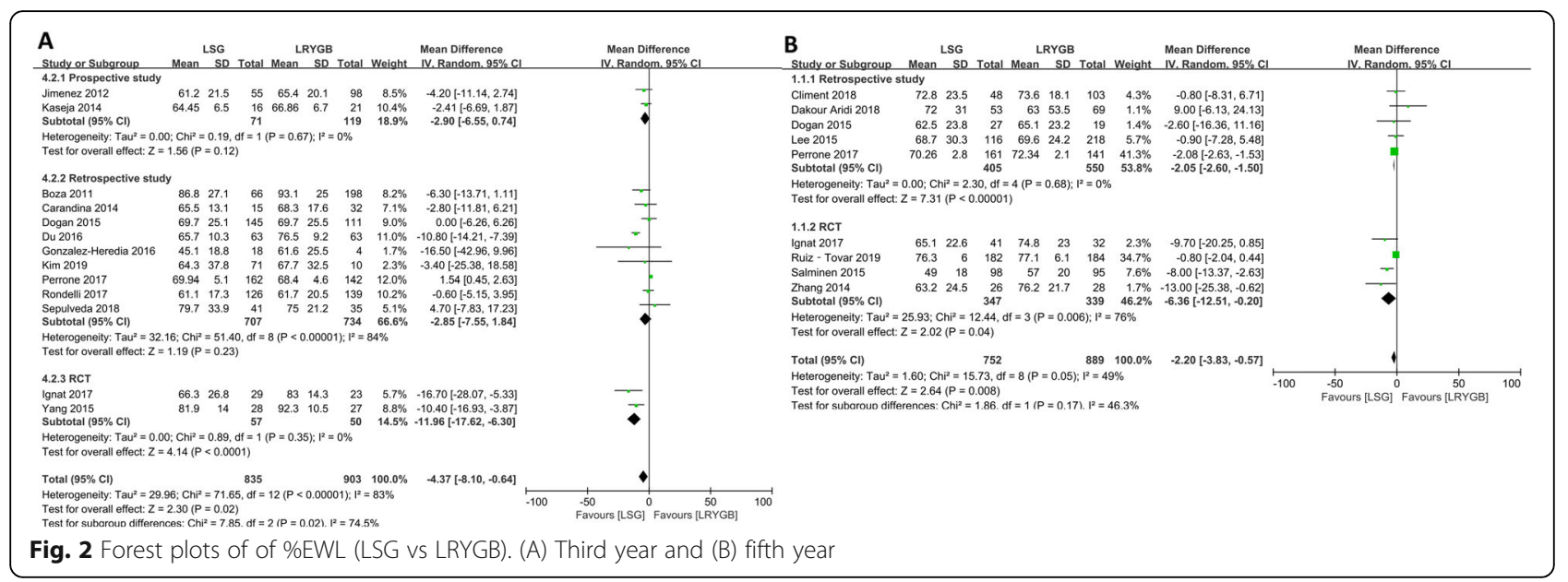

retrospective studies and RCTs. At 5-year follow-up, no statistical difference was noted among all prospective studies, retrospective studies, or RCTs. Details are shown in Fig. 3.

\section{Hypertension}

Eleven articles focused on hypertension representing 1456 patients (694 in the LSG group, 762 in the LRYGB group). No statistical difference was observed in hypertension at the 3-year follow-up and all subgroup analyses between LRYGB and LSG. Interestingly, compared with the LSG group, the LRYGB group has higher remission rate of hypertension during the fifth postoperative year $(\mathrm{OR}=0.51,95 \% \mathrm{CI}=$ $0.38-0.68, p<0.001$, fixed-effects model). In the subgroup analysis, the outcomes of LRYGB were better than those of LSG among all prospective studies, retrospective studies, and RCTs (Fig. 4).

\section{Dyslipidemia}

Although no statistical difference in remission of dyslipidemia at 3-year follow-up was found, LRYGB had higher remission rate of dyslipidemia at 5-year follow-up $(\mathrm{OR}=0.30,95 \% \mathrm{CI}=0.19-0.48, p<0.001$, fixed-effects model). The remission rate of abnormally low-density lipoprotein (LDL) at 5-year follow-up resolved considerably more common in the LRYGB group than in the LSG group $(\mathrm{OR}=0.27,95 \% \mathrm{CI}=0.11-0.68, p=0.006$, fixed-effects model). Moreover, we did not find significant difference in the treatment effect between the two groups in terms of high-density lipoprotein and triglycerides in the fifth year of follow-up (Fig. 5).

\section{Sensitivity analysis and publication bias}

In each group analysis, we excluded each study and the overall effect was consistent. Begg's and Egger's test were performed on the results of each group, and the results showed no publication bias $(p>0.05)$.

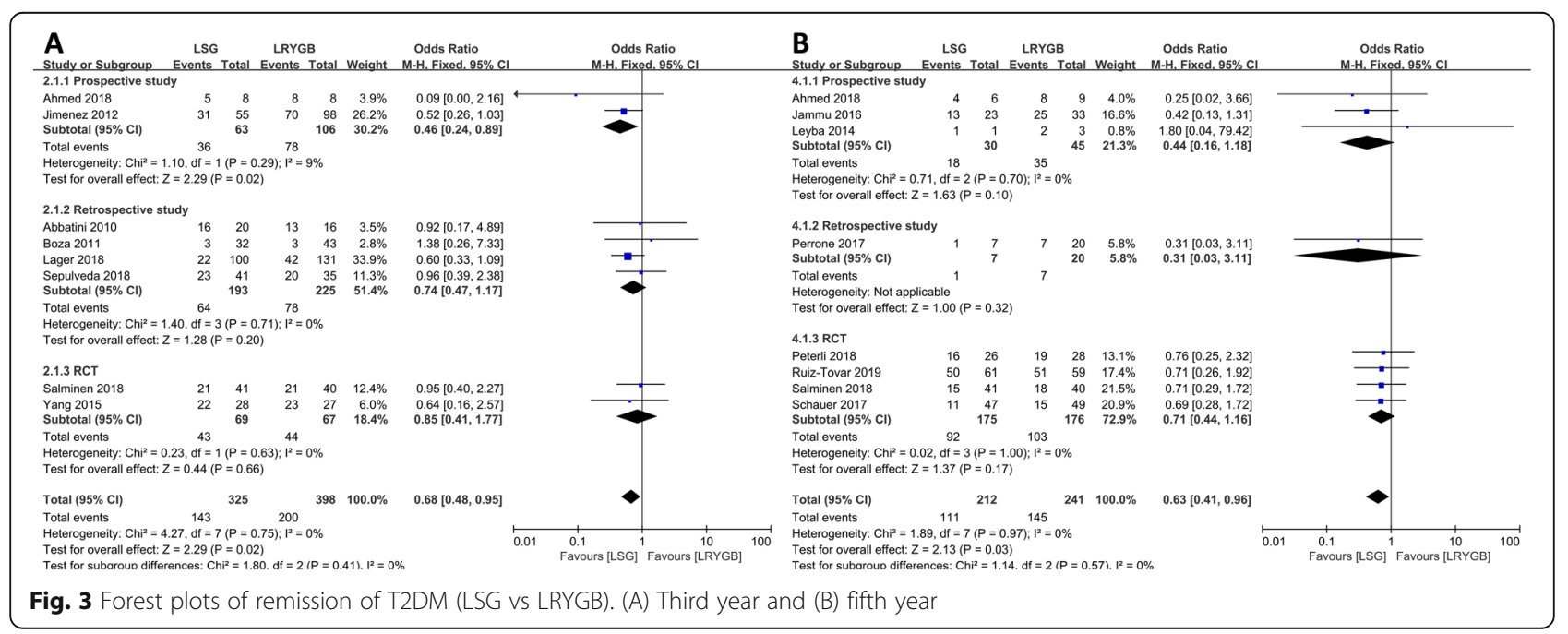




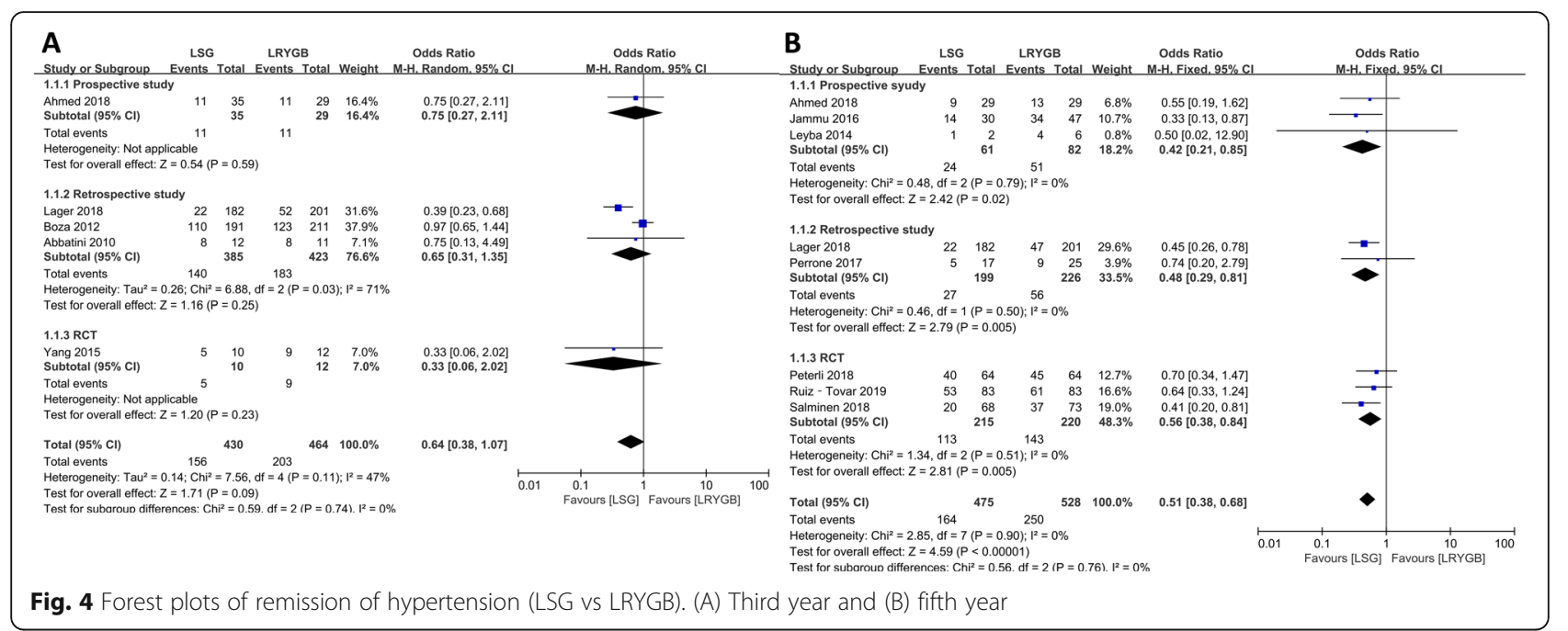

\section{Discussion}

LSG and LRYGB are the most commonly performed bariatric surgeries in the last decade. They are not only safe and effective for weight loss, but also have a role in alleviating complications. This meta-analysis was based on 27 multi-screened, high-quality highly reviewed articles. The main results of this metaanalysis were as follows: (1) no significant difference was found in \%EWL and remission of hypertension and dyslipidemia between the LSG group and the LRYGB group at 3-year follow-up. (2) Five-year follow-up results showed that LRYGB had an advantage over LSG in terms of \%EWL, remission of hypertension, dyslipidemia, and abnormally LDL. (3) The remission rate of T2DM was superior in LSG in both 3- and 5-year follow-up.

\section{\%EWL}

Our mid-term (3-year follow-up) results showed that both groups had good outcomes and that there was no significant difference in \%EWL, which might be attributed to a lower initial body mass index (BMI) [37]. Hosam et al. observed that the preoperative BMI had a strong impact on the final outcome of LSG [38]. Interestingly, LRYGB gradually showed its advantages during the 5-year follow-up period, which agreed with the research conclusions of Zhang et al. [6]. As a restrictive procedure, high-calorie and high-sugar foods after LSG could cause rebound weight gain, so patients should strictly follow the postoperative nutritional guidelines. LSG reduces weight by limiting calories, but expanding sleeved stomach caused by dietary failure will lead to weight recovery. Many articles indicated that LRYGB

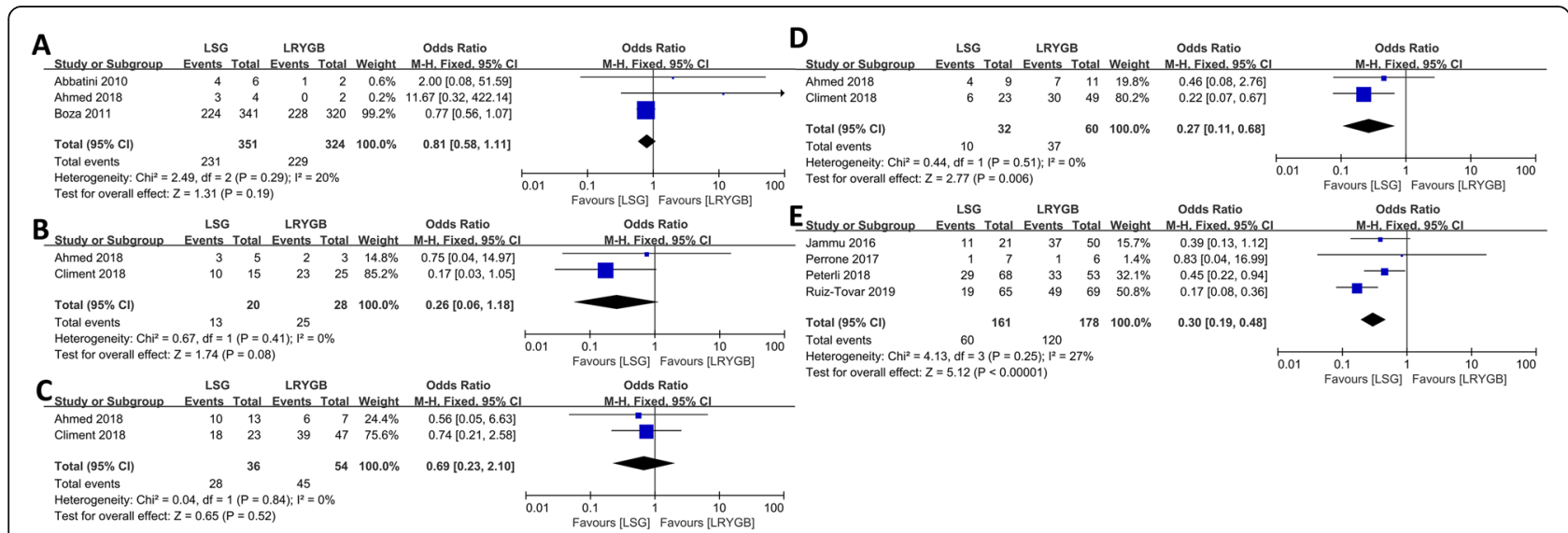

Fig. 5 Forest plots of remission of dyslipidemia (LSG vs LRYGB). (A) Remission of dyslipidemia in the third year, (B) remission of high triglycerides in the fifth year, (C) remission of low HDL in the fifth year, (D) remission of high LDL in the fifth year, and (E) remission of dyslipidemia in the fifth year 
was superior to LSG in weight reduction especially for super-obese patients $[3,6]$. Thus, LSG might be converted to LRYGB due to insufficient weight loss or severe reflux esophagitis $[5,27]$.

Nowadays, the goal of bariatric surgery is not as simple as controlling weight alone. It is equally important to improve complications such as T2DM, dyslipidemia, hypertension, obstructive sleep apnea, liver, and kidney function damage [39]. Bariatric surgery could also be used as potential treatment of metabolic syndrome, and the concept of "metabolic surgery" was born [40].

\section{T2DM}

The results of the 3-year and 5-year follow-up showed that the T2DM remission rate of LRYGB was higher than that of LSG. The heterogeneity of the results was very low, and data were highly feasible. Jiménez et al. showed that more than half of patients who underwent LSG bariatric surgery had a T2DM remission after 3 years of follow-up, and this goal was achieved with only 2 years in the LRYGB group. T2DM will recur after a long time, which results in decreased remission rate; therefore, long-term follow-up is more meaningful [25]. Some research results showed that \%EWL was a determinant of T2DM remission. To determine the effect of fasting insulin and glucose reduction, the degree of \%EWL was more significant than the type of surgery. This may be the reason why LRYGB was more effective than LSG as T2DM treatment [16].

Many studies had shown that hormonal changes also play an important role in weight loss and remission of metabolic disease after surgery. Several theories about hormonal changes were intensively under investigation, but none of them currently stand out as the leading mechanism. According to previous studies, LRYGB and LSG induce similar changes in these hormones expect for ghrelin. Ghrelin is reduced after LSG as large parts of the stomach were resected, whereas ghrelin may increase or remain stable after LRYGB [41, 42]. A recent study showed that LRYGB was characterized by accelerated absorption of glucose and amino acids, whereas protein metabolism after LSG did not differ significantly from controls, suggesting that different mechanisms explain improved glycemic control and weight loss after these surgical procedures [43].

\section{Hypertension}

Obesity has become one of the most important causes of hypertension, as 60 to $70 \%$ of hypertension in adults can be attributed to obesity [44]. In this study, no statistical difference in midterm hypertension remission rates was found between the two groups, but long-term results showed that LRYGB had an obvious advantage. The results of these studies were similar to our results $[2,10$,
15, 20]. The exact mechanism of hypertension and a range of cardiovascular diseases due to obesity have not been confirmed. However, the neuroendocrine system and adipokines were thought to play a leading role. Obesity-related hypertension was also thought to be associated with metabolic syndrome related to glucose intolerance [44, 45]. Previous studies had shown that LRYGB may be the first choice for obese patients with cardiovascular risk [46].

\section{Dyslipidemia}

Compared to LSG, LRYGB was a more effective treatment of dyslipidemia [37], but the two groups had similar effects on obesity metabolic disorders [17]. Studies that combined various factors showed that the remission rate of abnormally LDL in LRYGB was higher than that in LSG [27, 32], because LRYGB might have reduced the absorption rate of LDL than did LSG [32]. Other studies have shown that LSG has no significant effect on the reduction of LDL, while LRYGB could cause absorption disorder in individuals; perhaps, this is the reason why LRYGB could improve the overall lipid profile better than LSG [47].

The advantages of LRYGB were as follows: The shortterm results of LRYGB might be as effective as those of LSG, but medium- or long-term results could show a clear advantage. LRYGB could ensure a good quality of life and minimum side effects, effectively control metabolic complications including T2DM and hypertension $[3,15]$, provide better blood glucose control, and lower blood lipid level effect [5].

The advantages of the study were a large population base, detailed subgroup analysis (based on follow-up time divided into 3 years (mid-term) and 5 years (long term), three types of trials, and clear indicators for recovery of complications (included only in remission). In a retrospective review of similar meta-analyses, results of Yang et al.'s meta-analysis were similar to the results of the present study in that LRYGB patients had significantly reduced their weight during the 3- or 5-year follow-up period compared to LSG patients [48]. However, they combined the improvement and remission of complications. Heterogeneity could be introduced because of the different definitions of improvement and remission. This might be the reason why LRYGB had not shown an advantage in treating comorbidities. Huang et al.'s meta-analysis showed that LSG and LRYGB had no significant advantage in short- or long-term blood glucose control, which is contrary to our conclusion, because their patients were followed at 1-2 years, and there are limited studies with 3- or 5-year follow-up periods. In addition, they did not perform subgroup analyses based on the type of trial, which was less convincing than this article [49]. 
Zhao et al. [50]. included 11 RCTs in their metaanalysis, and the results showed no difference in \%EWL and T2DM between the two surgical methods (LSG and LRYGB), which may be attributed to the lack of longterm follow-up data, with only 3 studies providing data of 3 years and 5 years respectively. Sharples [51] included 5 RCTs and concluded demonstrated a significantly greater \%EWL in patients undergoing LRYGB compared with LSG. However, there was no significant difference between LRYGB and LSG in rates of resolution or improvement of diabetes. Similarly, HbA1C levels were not significantly different between the two procedures. A meta-analysis involving 33 RCTs showed that LRYGB resulted in greater BMI loss at 1 and 3 years; however, there was insufficient randomized evidence to draw any conclusions regarding weight loss between the 2 procedures at 5 years. No differences between the two procedures were found in remission of type 2 diabetes, despite a trend at every time interval favoring LRYGB, hypertension [52]. However, most of the studies included in this study were short-term follow-up data with high heterogeneity, so the results need careful interpretation.

This study has some limitations. This study included retrospective studies that reduced the overall quality of evidence. We were unable to classify obese people according to the degree of obesity, because it was not explicitly mentioned in the papers. In addition, most of the countries included were Western and were not necessarily suitable for the Asian population. Finally, the criteria for incorporating obese patients were different because varied literature types were included. These deficiencies will affect the scope and accuracy of the findings of this meta-analysis.

\section{Conclusion}

In summary in terms of the long-term effects of bariatric surgery, including \%EWL and the remission of complications (T2DM, hypertension and dyslipidemia), the effect of LRYGB was better than of LSG.

\section{Additional File}

Additional file $\mathbf{1}$ Table S1 Quality assessment of studies included.

\author{
Abbreviations \\ BMI: Body mass index; EWL: Percentage of excess weight loss; LDL: Low- \\ density lipoprotein, 95\%Cl: 95\% confidence intervals; LRYGB: Laparoscopic \\ Roux-en-Y gastric bypass; LSG: Laparoscopic sleeve gastrectomy; \\ NOS: Newcastle-Ottawa Quality Assessment Scale; OR: Odds ratio; \\ PRISMA: Preferred Reporting Items for Systematic Reviews and Meta- \\ Analyses, \%; RCT: Randomized controlled trial; T2DM: Type 2 diabetes \\ mellitus; WMD: Weighted mean difference
}

\section{Author's contributions}

Each author contributed significantly to concept and development of the present paper. $L G$ and PC designed this study. $L G, X H, S L, D M$, and ZS collected the data. $\mathrm{LG}$ and $\mathrm{XH}$ analyzed the data. SL and DM interpreted the data. ZS, PAK and LG drafted the manuscript. LG and DMN interpreted and revised the manuscript. All authors have read and approved the manuscript.

\section{Funding}

The authors have no financial support to declare.

\section{Availability of data and materials}

All data generated or analysed during this study are included in this published article.

\section{Ethics approval and consent to participate}

This article does not contain any studies with human or animal subjects performed by any of the authors.

\section{Consent for publication}

Not applicable.

\section{Competing interests}

The authors declare that they have no conflict of interest.

\section{Author details}

${ }^{1}$ Department of General Surgery, HwaMei Hospital, University of Chinese Academy of Sciences, Northwest Street 41, Haishu District, Ningbo, Zhejiang 315010, China. ${ }^{2}$ The Second Clinical Medical College, Zhejiang Chinese Medical University, Hangzhou, Zhejiang, China. ${ }^{3}$ Basic Medical College, Zhejiang Chinese Medical University, Hangzhou, Zhejiang, China. ${ }^{4}$ Department of General Surgery, Zhejiang University School of Medicine Sir Run Run Shaw Hospital, Hangzhou, Zhejiang, China. ${ }^{5}$ Monash University School of Public Health and Preventive Medicine, Melbourne, Australia.

${ }^{6}$ Medical College of Ningbo University, Hangzhou, Zhejiang, China.

Received: 27 November 2019 Accepted: 7 February 2020

Published online: 12 February 2020

\section{References}

1. Abbatini F, Rizzello M, Casella G, Alessandri G, Capoccia D, Leonetti F, Basso N. Long-term effects of laparoscopic sleeve gastrectomy, gastric bypass, and adjustable gastric banding on type 2 diabetes. Surg Endosc. 2010;24:1005-10.

2. Du X, Zhang SQ, Zhou HX, Li X, Zhang XJ, Zhou ZG, Cheng Z. Laparoscopic sleeve gastrectomy versus Roux-en-Y gastric bypass for morbid obesity: a 1:1 matched cohort study in a Chinese population. Oncotarget. 2016;7:76308-15.

3. Ignat M, Vix M, Imad I, D'Urso A, Perretta S, Marescaux J, Mutter D. Randomized trial of Roux-en-Y gastric bypass versus sleeve gastrectomy in achieving excess weight loss. Br J Surg. 2017;104:248-56.

4. Dakour Aridi H, Khazen G, Safadi BY. Comparison of outcomes between laparoscopic Roux-en-Y gastric bypass and sleeve Gastrectomy in a Lebanese bariatric surgical practice. Obes Surg. 2018;28:396-404.

5. Dogan K, Gadiot RP, Aarts EO, Betzel B, van Laarhoven CJ, Biter LU, Mannaerts GH, Aufenacker TJ, Janssen IM, Berends FJ. Effectiveness and safety of sleeve Gastrectomy, gastric bypass, and adjustable gastric banding in morbidly obese patients: a multicenter, retrospective, matched cohort study. Obes Surg. 2015;25:1110-8.

6. Zhang Y, Zhao H, Cao Z, Sun X, Zhang C, Cai W, Liu R, Hu S, Qin M. A randomized clinical trial of laparoscopic Roux-en-Y gastric bypass and sleeve gastrectomy for the treatment of morbid obesity in China: a 5-year outcome. Obes Surg. 2014;24:1617-24.

7. Vidal P, Ramon JM, Goday A, Benaiges D, Trillo L, Parri A, Gonzalez S, Pera M, Grande L. Laparoscopic gastric bypass versus laparoscopic sleeve gastrectomy as a definitive surgical procedure for morbid obesity. Mid-term results Obes Surg. 2013;23:292-9.

8. Li J, Lai D, Wu D. Laparoscopic Roux-en-Y gastric bypass versus laparoscopic sleeve Gastrectomy to treat morbid obesity-related comorbidities: a systematic review and meta-analysis. Obes Surg. 2016;26:429-42.

9. Lakdawala MA, Bhasker A, Mulchandani D, Goel S, Jain S. Comparison between the results of laparoscopic sleeve gastrectomy and laparoscopic Roux-en-Y gastric bypass in the Indian population: a retrospective 1 year study. Obes Surg. 2010;20:1-6. 
10. Leyba JL, Llopis SN, Aulestia SN. Laparoscopic Roux-en-Y gastric bypass versus laparoscopic sleeve gastrectomy for the treatment of morbid obesity. A prospective study with 5 years of follow-up. Obes Surg. 2014;24:2094-8.

11. Alexandrou A, Armeni E, Kouskouni E, Tsoka E, Diamantis T, Lambrinoudaki I. Cross-sectional long-term micronutrient deficiencies after sleeve gastrectomy versus Roux-en-Y gastric bypass: a pilot study. Surg Obes Relat Dis. 2014;10:262-8.

12. Coupaye M, Gorbatchef C, Calabrese D, Sami O, Msika S, Coffin B, Ledoux S. Gastroesophageal reflux after sleeve Gastrectomy: a prospective mechanistic study. Obes Surg. 2018;28:838-45.

13. Kehagias I, Karamanakos SN, Argentou M, Kalfarentzos F. Randomized clinical trial of laparoscopic Roux-en-Y gastric bypass versus laparoscopic sleeve gastrectomy for the management of patients with $\mathrm{BMI}<50 \mathrm{~kg} / \mathrm{m} 2$. Obes Surg. 2011;21:1650-6.

14. Ruiz-Tovar J, Carbajo MA, Jimenez JM, Castro MJ, Gonzalez G, Ortiz-deSolorzano J, Zubiaga L. Long-term follow-up after sleeve gastrectomy versus Roux-en-Y gastric bypass versus one-anastomosis gastric bypass: a prospective randomized comparative study of weight loss and remission of comorbidities. Surg Endosc. 2019;33:401-10.

15. Perrone F, Bianciardi E, Ippoliti S, Nardella J, Fabi F, Gentileschi P. Long-term effects of laparoscopic sleeve gastrectomy versus Roux-en-Y gastric bypass for the treatment of morbid obesity: a monocentric prospective study with minimum follow-up of 5 years. Updat Surg. 2017;69:101-7.

16. Pucci A, Tymoszuk U, Cheung WH, Makaronidis JM, Scholes S, Tharakan G, Elkalaawy M, Guimaraes M, Nora M, Hashemi M, Jenkinson A, Adamo M, Monteiro MP, Finer N, Batterham RL. Type 2 diabetes remission 2 years post Roux-en-Y gastric bypass and sleeve gastrectomy: the role of the weight loss and comparison of DiaRem and DiaBetter scores. Diabet Med. 2018:35:360-7.

17. Yang J, Wang C, Cao G, Yang W, Yu S, Zhai H, Pan Y. Long-term effects of laparoscopic sleeve gastrectomy versus roux-en-Y gastric bypass for the treatment of Chinese type 2 diabetes mellitus patients with body mass index 28-35 kg/m (2). BMC Surg. 2015;15:88.

18. Jammu GS, Sharma R. A 7-year clinical audit of 1107 cases comparing sleeve Gastrectomy, Roux-en-Y gastric bypass, and mini-gastric bypass, to determine an effective and safe bariatric and metabolic procedure. Obes Surg. 2016;26:926-32.

19. Lager CJ, Esfandiari NH, Luo Y, Subauste AR, Kraftson AT, Brown MB, Varban OA, Meral R, Cassidy RB, Nay CK, Lockwood AL, Bellers D, Buda CM, Oral EA. Metabolic Parameters, Weight Loss, and Comorbidities 4 Years After Rouxen-Y Gastric Bypass and Sleeve Gastrectomy. Obes Surg. 2018;28:3415-23.

20. Salminen $P$, Helmio M, Ovaska J, Juuti A, Leivonen M, Peromaa-Haavisto $P$, Hurme S, Soinio M, Nuutila P, Victorzon M. Effect of laparoscopic sleeve Gastrectomy vs laparoscopic Roux-en-Y gastric bypass on weight loss at 5 years among patients with morbid obesity: the SLEEVEPASS randomized clinical trial. Jama. 2018;319:241-54.

21. Moher D, Shamseer L, Clarke M, Ghersi D, Liberati A, Petticrew M, Shekelle P, Stewart LA. Preferred reporting items for systematic review and metaanalysis protocols (PRISMA-P) 2015 statement. Syst Rev. 2015;4:1.

22. Green S, Higgins JPT, Alderson P, et al. Cochrane handbook for systematic reviews of interventions version 5.1.0 [updated March 2011]. The Cochrane Collaboration, 2011.

23. Stang A. Critical evaluation of the Newcastle-Ottawa scale for the assessment of the quality of nonrandomized studies in meta-analyses. Eur J Epidemiol. 2010;25:603-5.

24. Boza C, Gamboa C, Salinas J, Achurra P, Vega A, Perez G. Laparoscopic Rouxen-Y gastric bypass versus laparoscopic sleeve gastrectomy: a case-control study and 3 years of follow-up. Surg Obes Relat Dis. 2012;8:243-9.

25. Jimenez A, Casamitjana R, Flores L, Viaplana J, Corcelles R, Lacy A, Vidal J. Long-term effects of sleeve gastrectomy and Roux-en-Y gastric bypass surgery on type 2 diabetes mellitus in morbidly obese subjects. Ann Surg. 2012;256:1023-9.

26. Carandina S, Maldonado PS, Tabbara M, Valenti A, Rivkine E, Polliand C, Barrat C. Two-step conversion surgery after failed laparoscopic adjustable gastric banding. Comparison between laparoscopic RouX-en-Y gastric bypass and laparoscopic gastric sleeve. Surg Obes Relat Dis. 2014;10:1085-91.

27. Lee WJ, Pok EH, Almulaifi A, Tsou JJ, Ser KH, Lee YC. Medium-term results of laparoscopic sleeve Gastrectomy: a matched comparison with gastric bypass. Obes Surg. 2015;25:1431-8.

28. Gonzalez-Heredia R, Sanchez-Johnsen L, Valbuena VS, Masrur M, Murphey M, Elli E. Surgical management of super-super obese patients: Roux-en-Y gastric bypass versus sleeve gastrectomy. Surg Endosc. 2016;30:2097-102.
29. Kaseja K, Majewski WD, Kolpiewicz B. A comparison of EFECTIVENESS, and an assessment of the quality of LIFE of patients after laparoscopic sleeve GAST RECTOMY and roux-en-y gastric bypass. Ann Acad Med Stetin. 2014;60:7-12.

30. Rondelli F, Bugiantella W, Vedovati MC, Mariani E, Balzarotti Canger RC, Federici S, Guerra A, Boni M. Laparoscopic gastric bypass versus laparoscopic sleeve gastrectomy: a retrospective multicenter comparison between early and longterm post-operative outcomes. Int J Surg. 2017;37:36-41.

31. Peterli R, Wolnerhanssen BK, Peters $T$, Vetter D, Kroll D, Borbely $Y$, Schultes B, Beglinger C, Drewe J, Schiesser M, Nett P, Bueter M. Effect of laparoscopic sleeve Gastrectomy vs laparoscopic Roux-en-Y gastric bypass on weight loss in patients with morbid obesity: the SM-BOSS randomized clinical trial. Jama. 2018;319:255-65.

32. Climent E, Benaiges D, Flores-Le Roux JA, Ramon JM, Pedro-Botet J, Goday A. Changes in the lipid profile 5 years after bariatric surgery: laparoscopic Roux-en-Y gastric bypass versus laparoscopic sleeve gastrectomy. Surg Obes Relat Dis. 2018;14:1099-105.

33. Sepulveda M, Alamo M, Preiss Y, Valderas JP. Metabolic surgery comparing sleeve Gastrectomy with Jejunal bypass and RouX-en-Y gastric bypass in type 2 diabetic patients after 3 years. Obes Surg. 2018;28:3466-73.

34. Ahmed B, King WC, Gourash W, Belle SH, Hinerman A, Pomp A, Dakin G, Courcoulas AP. Long-term weight change and health outcomes for sleeve gastrectomy (SG) and matched Roux-en-Y gastric bypass (RYGB) participants in the longitudinal assessment of bariatric surgery (LABS) study. Surgery. 2018;164:774-83.

35. Kim G, Tan CS, Tan KW, Lim SPY, So JBY, Shabbir A. Sleeve Gastrectomy and Roux-en-Y gastric bypass Lead to comparable changes in body composition in a multiethnic Asian population. J Gastrointest Surg. 2019;23:445-50.

36. Schauer PR, Bhatt DL, Kirwan JP, Wolski K, Aminian A, Brethauer SA, Navaneethan SD, Singh RP, Pothier CE, Nissen SE, Kashyap SR. Bariatric surgery versus intensive medical therapy for diabetes - 5-year outcomes. N Engl J Med. 2017;376:641-51.

37. El Chaar M, Hammoud N, Ezeji G, Claros L, Miletics M, Stoltzfus J. Laparoscopic sleeve gastrectomy versus laparoscopic Roux-en-Y gastric bypass: a single center experience with 2 years follow-up. Obes Surg. 2015; 25:254-62.

38. Elbanna H, Ghnnam W, Negm A, Youssef T, Emile S, El Metwally T, Elalfy K. Impact of preoperative body mass index on the final outcome after laparoscopic sleeve gastrectomy for morbid obesity. Ulus Cerrahi Derg. 2016;32:238-43.

39. Zhang N, Maffei A, Cerabona T, Pahuja A, Omana J, Kaul A. Reduction in obesity-related comorbidities: is gastric bypass better than sleeve gastrectomy? Surg Endosc. 2013;27:1273-80.

40. Vix M, Diana M, Liu KH, D'Urso A, Mutter D, Wu HS, Marescaux J. Evolution of glycolipid profile after sleeve gastrectomy vs. Roux-en-Y gastric bypass: results of a prospective randomized clinical trial. Obes Surg. 2013;23:613-21.

41. Batterham RL, Cummings DE. Mechanisms of diabetes improvement following bariatric/metabolic surgery. Diabetes Care. 2016;39:893-901.

42. Ryan KK, Tremaroli V, Clemmensen C, Kovatcheva-Datchary P, Myronovych A, Karns R, Wilson-Perez HE, Sandoval DA, Kohli R, Backhed F, Seeley RJ. FXR is a molecular target for the effects of vertical sleeve gastrectomy. Nature. 2014:509:183-8.

43. Svane MS, Bojsen-Moller KN, Martinussen C, Dirksen C, Madsen JL, Reitelseder S, Holm L, Rehfeld JF, Kristiansen VB, van Hall G, Holst J J, Madsbad S. Postprandial Nutrient Handling and Gastrointestinal Hormone Secretion After Roux-en-Y Gastric Bypass vs Sleeve Gastrectomy. Gastroenterology. 2019;156:1627-1641.e1621.

44. Kotchen TA. Obesity-related hypertension: epidemiology, pathophysiology, and clinical management. Am J Hypertens. 2010;23:1170-8.

45. Narkiewicz K. Obesity and hypertension--the issue is more complex than we thought. Nephrol Dial Transplant. 2006;21:264-7.

46. Peterli $R$, Wolnerhanssen BK, Vetter $D$, Nett $P$, Gass $M$, Borbely $Y$, Peters $T$, Schiesser M, Schultes B, Beglinger C, Drewe J, Bueter M. Laparoscopic sleeve Gastrectomy versus Roux-Y-gastric bypass for morbid Obesity-3-year outcomes of the prospective randomized Swiss multicenter bypass or sleeve study (SM-BOSS). Ann Surg. 2017;265:466-73.

47. Benaiges D, Flores-Le-Roux JA, Pedro-Botet J, Ramon JM, Parri A, Villatoro M, Carrera MJ, Pera M, Sagarra E, Grande L, Goday A. Impact of restrictive (sleeve gastrectomy) vs hybrid bariatric surgery (Roux-en-Y gastric bypass) on lipid profile. Obes Surg. 2012;22:1268-75.

48. Yang P, Chen B, Xiang S, Lin XF, Luo F, Li W. Long-term outcomes of laparoscopic sleeve gastrectomy versus RouX-en-Y gastric bypass for morbid 
obesity: results from a meta-analysis of randomized controlled trials. Surg Obes Relat Dis. 2019;15:546-55.

49. Huang $X$, Liu T, Zhong M, Cheng Y, Hu S, Liu S. Predictors of glycemic control after sleeve gastrectomy versus Roux-en- $Y$ gastric bypass: a metaanalysis, meta-regression, and systematic review. Surg Obes Relat Dis. 2018; 14:1822-31.

50. Zhao H, Jiao L. Comparative analysis for the effect of Roux-en-Y gastric bypass vs sleeve gastrectomy in patients with morbid obesity: evidence from 11 randomized clinical trials (meta-analysis). Int J Surg. 2019;72:216-23.

51. Sharples AJ, Mahawar K. Systematic Review and Meta-Analysis of Randomised Controlled Trials Comparing Long-Term Outcomes of Roux-EnY Gastric Bypass and Sleeve Gastrectomy. Obes Surg. 2020;30:664-72.

52. Lee Y, Doumouras AG, Yu J, Aditya I, Gmora S, Anvari M, Hong D. Laparoscopic sleeve Gastrectomy versus laparoscopic Roux-en-Y gastric bypass: a systematic review and meta-analysis of weight loss, comorbidities, and biochemical outcomes from randomized controlled trials. Ann Surg 2019;23:1-15.

\section{Publisher's Note}

Springer Nature remains neutral with regard to jurisdictional claims in published maps and institutional affiliations.

Ready to submit your research? Choose BMC and benefit from:

- fast, convenient online submission

- thorough peer review by experienced researchers in your field

- rapid publication on acceptance

- support for research data, including large and complex data types

- gold Open Access which fosters wider collaboration and increased citations

- maximum visibility for your research: over $100 \mathrm{M}$ website views per year

At BMC, research is always in progress.

Learn more biomedcentral.com/submissions 